Rev. Téc. Ing. Univ. Zulia. Vol. 43, No. 1, 2020, 12-18

\title{
Modification of a hot asphalt mix by dry process using special waste (slag) from car batteries
}

\author{
Wilson Cando $^{1 *}$, Gustavo Yánez ${ }^{1}$, Patricio Castro ${ }^{1}$, Slendi M. Constante ${ }^{1}$, Lenys Fernández ${ }^{2,3}$ (D), \\ Patricio Espinoza-Montero ${ }^{2}$ (D)
}

\author{
${ }^{1}$ Pontificia Universidad Católica del Ecuador, Facultad de Ingeniería, Avenida 12 de octubre y Roca, Apartado \\ 17-01-2184, Quito-Ecuador \\ ${ }^{2}$ Pontificia Universidad Católica del Ecuador, Facultad de Ciencias Exactas y Naturales, Escuela de Ciencias \\ Químicas, Avenida 12 de octubre y Roca, Apartado 17-01-2184, Quito, Ecuador \\ ${ }^{3}$ Universidad Simón Bolívar, Departamento de Química, Apartado 89000, Caracas-Venezuela \\ *Autor de Contacto: WOCANDO@puce.edu.ec \\ https://doi.org/10.22209/rt.v43n1a02
}

Recepción: 12/02/2019 | Aceptación: 12/07/2019 | Publicación: 20/12/2019

\begin{abstract}
The characterization of a modified asphalt mixture with special waste (slag) from car batteries is reported in the present work. The modification was made through the dry process and the relationship of voids that characterizes the quality of an asphalt mixture in the modified and unmodified material was evaluated. The study was conducted by adding 5\%, 15\%, 25\% and 35\% slag percentages to the asphalt mix. The results obtained from integrating the special waste into the mixture indicate that this modification improves its mechanical properties and the performance of the materials when it is used in the mixture. Compared to the unmodified mixture the modified sample, it showed an increase of $30 \%$ in stability and 8\% in flow, which predicts an improvement in the mechanical properties of the mixture in terms of increased resistance. On the other hand, the volumetric properties comply with the standard requirements, and the rigidity modulus test, that determined the behavior of the pavement on site,also increased, which demonstrates the advantages of using this slag residue in the asphalt mix.
\end{abstract}

Key words: Waste of Automobile Batteries; Modified Asphalt Mix; Asphalt-Slag mixture.

\section{Modificación de una mezcla asfáltica en caliente mediante procedimiento en seco utilizando desechos especiales (Escoria) proveniente de baterías de automóviles}

\begin{abstract}
Resumen
Se reporta la caracterización de una mezcla asfáltica, modificada con desechos especiales proveniente de baterías de automóviles. La modificación se realizó por vía seca y se evaluó la relación de vacíos que caracteriza la calidad de una mezcla asfáltica, en el material modificado y sin modificar. El estudio se realizó añadiendo en peso porcentajes de 5\%, 15\%, $25 \%$ y $35 \%$ de escoria a la mezcla asfáltica. Los resultados obtenidos de la integración de desechos especiales a la mezcla, indican que dicha modificación mejora sus propiedades mecánicas y el rendimiento de los materiales al momento de ser usados en la mezcla. En comparación con la mezcla sin modificar, la muestra modificada presentó un aumento de un $30 \%$ en estabilidad y un $8 \%$ en flujo, lo que predice una mejora en las propiedades mecánicas de la mezcla en cuanto a aumento en resistencia. Por otro lado, las propiedades volumétricas de la mezcla modificada se mantuvieron en el rango dispuesto en la norma y el ensayo de módulo de rigidez, que determinó el comportamiento del pavimento en sitio, igualmente aumentó. Lo que demuestra las ventajas de modificar la mezcla asfáltica con la escoria de baterías desechadas.
\end{abstract}

Palabras clave: Desechos de Baterías de Automóviles; Mezcla Asfáltica Modificada; Mezcla Asfalto-Escoria. 


\section{Introducción}

En la actualidad el impacto ambiental que ha generado los desechos de baterías de automóviles usados, se evidencia por los altos daños al medio ambiente y a la salud de seres vivos [1-3]. El reciclaje de baterías desechadas, es un hábito que están adquiriendo las empresas ecuatorianas productoras de este dispositivo indispensable para que un automóvil pueda funcionar. Según estadísticas [4] al menos 100 millones de baterías al año llegan a su vida útil, un $90 \%$ es utiliza para producir nuevas baterías y un $10 \%$ son depositadas en pozos considerados como botaderos o basurero de baterías y en otros casos en terrenos baldíos, acequias, caminos vecinales, causes de agua, etc. Los daños que pueden causar a las personas que están en contacto con este desecho, ha hecho que las empresas tomen opciones de reutilizarlo, mediante el análisis de tratamiento anual de desechos sólidos de acuerdo a las normas establecidas para su reutilización. Una de las opciones para aprovechar este material es incorporarlo a la construcción [5], por lo que la siguiente investigación se enfocó en estudiar la inclusión de este tipo de desecho en mezclas asfálticas convencionalmente utilizadas en Ecuador en la construcción de carreteras. Una mezcla asfáltica está constituida por un material pétreo recubierto con una película de asfalto [6], uniformemente combinados en proporciones específicas. Las cantidades relativas de estos materiales, determinan las propiedades y características de la mezcla [7]. Específicamente se reporta la modificación de la mezcla asfáltica, reemplazando agregado por desechos especiales (Escoria) proveniente de baterías de automóviles en el tamaño de agregado equivalente a lo retenido en el tamiz número 8 , que según la normativa ASTM es de 2,36 milímetros. Se utilizó el método de diseño Marshall (AASHTO T 245 o ASTM D 15559), comúnmente utilizado en Latinoamérica para determinar las proporciones apropiadas de asfalto y agregado en una mezcla asfáltica en caliente. El proceso se realizó por vía seca, donde el material modificador se mezcla con los agregados, antes de adicionar el cemento asfáltico. En este caso, los desechos de escoria se suman como un sustituto de una pequeña parte del agregado fino. Se determinó el mejor método de mezcla para la modificación del pavimento con el porcentaje óptimo de escoria. Como sustituto de material pétreo, la escoria es un material de diferentes características físicas a las de los agregados, por lo que se establecieron datos comparativos de las propiedades físicas de este material como agregado en el asfalto modificado, y así determinar si estos cumplen con los requerimientos establecidos por las normativas ASTM, INEN Y AASHTO. Se pretende lograr pavimentos con mejor desempeño que los que se utilizan actualmente. La modificación de una mezcla asfáltica con desechos de este tipo es de vanguardia, aunque hay mezclas modificadas con residuos de "pet", esta escoria resulto con beneficios tanto para la mezcla asfáltica como para el medio ambiente. Fue de gran importancia lograr en la mezcla modificada el aumento de la estabilidad, así como el módulo de rigidez, por lo que la durabilidad será mayor. Los resultados obtenidos indican que la mezcla modificada, resulta en una mezcla asfáltica cerrada con propiedades mejoradas.

\section{Materiales y Métodos}

Los agregados y asfalto utilizados, se obtuvieron de la planta Asfáltica Naranjo Ordoñez, Quito-Ecuador. Agregados gruesos 3/4" $(1,905 \mathrm{~cm})$ y $1 / 2$ " $(1,27 \mathrm{~cm})$, agregado fino $1 / 4 "$ " $(0,635 \mathrm{~cm})$, Mina Eduardo, ubicada en la rivera del Rio Pita en el cantón Rumiñahui, los desechos especiales fueron facilitados por la empresa Baterías Ecuador Fabribat CIA LTDA. Reactivos: TQ Orange clean 90 (Spartan), desmoldante (Talco + glicerina (Sigma-Aldrich), sulfato de magnesio (Sigma-Aldrich), ácido hidroclórico 37\% (Sigma-Aldrich), óxido rojizo (Sigma-Aldrich) y propanol 99\% (Sigma-Aldrich). Equipos: controlador RTF, modelo CS 325-B, James Cox \& Sons INC, regulador de vacío digital modelo $\mathrm{H}-1$, equipo Marshall $\mathrm{H}-1322$, Humboldt MFG. CO. Schiller Park IL 60176, modelo No. H-1782, horno Despatch Industries, máquina de prueba de cribamiento de ajuste manual Gilson, modelo TS-2, Gilson Company INC., tamiz vibrador de cribado, modelo 14860, W. S. Tyler, Viscosímetro DV-II + Pro, tanque de prueba de ductilidad, Soiltest INC, máquina universal HYD 25, Cooper Technology, compactador Marshall, modelo H-1336, Humboldt MFG. CO, estufa Universal 32 lt, modelo UNB-200, Memmert, máquina de abrasión los Ángeles, modelo 42-5305, Soiltest INC.

\section{Caracterización de agregados pétreos}

En la Tabla 1, se muestra los ensayos realizados a los materiales con su respectiva Normas y comprobación de las especificaciones establecidas. La verificación del cumplimiento de las especificaciones establecidas en las normas para el material bituminoso AC-20 obtenida en el laboratorio, se muestran en la Tabla 2.

\section{Caracterización de los desechos especiales (Escoria) proveniente de baterías de automóviles}

La escoria utilizada proviene del proceso de fundición del plomo obtenido de las baterías usadas en mezcla con aditivos como carbón, viruta de hierro y sosa cáustica. El proceso de reciclaje utilizado fue el siguiente. Trituración y separación: apertura de cajas, rotura y separación de componentes; para aislar los diferentes materiales que componen las baterías. Extracción de electrolito: se extrajeron los tapones de la batería y el electrolito por decantación; este último se almacenó en un tanque colector resistente al ataque del ácido y se neutralizó mediante el uso de carbonato de calcio $\left(\mathrm{CaCO}_{3}\right)$. Rotura de Baterías: una vez separado el ácido de las baterías, estas se cortaron, separando la tapa y carcasa y así sacar los insertos y placas de plomo (Scrap). Separación de insertos y pasta de plomo: cuando las baterías estuvieron fragmentadas, se separaron los 
Tabla 1. Caracterización de agregados pétreos

\begin{tabular}{|c|c|c|c|c|c|c|}
\hline \multirow{2}{*}{ Ensayo } & \multicolumn{3}{|c|}{ Norma } & \multirow{2}{*}{ Resultados } & \multirow{2}{*}{ Especificación } & \multirow{2}{*}{ Verificación } \\
\hline & INEN & AAS THO & ASTM & & & \\
\hline Equivalente de arena & ------ & $\mathrm{T}-176$ & D-2419 & $78 \%$ & Mayor a $50 \%$ & Cumple \\
\hline Desgaste & $86-860$ & $\mathrm{~T}-96$ & C-131 & $28,33 \%$ & Menor a $40 \%$ & Cumple \\
\hline Durabilidad & 863 & $\mathrm{~T}-104$ & C-88 & $5,60 \%$ & Max. $12 \%$ & Cumple \\
\hline $\begin{array}{l}\text { Gravedad Bulk, } \\
\text { agregado grueso }\end{array}$ & 857 & $\mathrm{~T}-85$ & C-128 & $2,51 \%$ & N/A & Cumple \\
\hline $\begin{array}{l}\text { Gravedad Bulk, } \\
\text { agregado intermedio }\end{array}$ & 857 & $\mathrm{~T}-85$ & C-128 & $2,45 \%$ & N/A & Cumple \\
\hline $\begin{array}{c}\text { Gravedad Bulk, } \\
\text { agregado fino }\end{array}$ & 856 & $\mathrm{~T}-84$ & C-127 & $2,46 \%$ & N/A & Cumple \\
\hline
\end{tabular}

N/A: No aplica

Tabla 2. Caracterización de cemento asfáltico AC-20

\begin{tabular}{|c|c|c|c|c|c|c|c|}
\hline \multirow{3}{*}{$\begin{array}{c}\text { Ensayo } \\
\text { Gravedad específica }\end{array}$} & \multicolumn{3}{|c|}{ Norma } & \multirow{3}{*}{$\begin{array}{c}\text { Resultados } \\
1,013\end{array}$} & \multirow{2}{*}{\multicolumn{2}{|c|}{ Especificaciones }} & \multirow{3}{*}{$\begin{array}{c}\text { Verificación } \\
\text { Cumple }\end{array}$} \\
\hline & \multirow{2}{*}{$\begin{array}{c}\text { INEN } \\
923\end{array}$} & \multirow{2}{*}{$\begin{array}{c}\text { AASTHO } \\
\mathrm{T}-43\end{array}$} & \multirow{2}{*}{$\begin{array}{c}\text { ASTM } \\
\text { D-70 }\end{array}$} & & & & \\
\hline & & & & & MOP001-T810.2,1 & MIN 1,0 & \\
\hline Penetración. (1/10mm) & ------ & $\mathrm{T}-49$ & D-5 & 70 & MOP001-T810.2,2 & MIN 60 & Cumple \\
\hline Índice de penetració & ------ & ----- & D3381 & $-0,5$ & ------ & $\begin{array}{l}\text { MIN }-1,5 \\
\text { MAX } 10\end{array}$ & Cumple \\
\hline $\begin{array}{c}\text { Punto de } \\
\text { reblandecimiento (oC) }\end{array}$ & 920 & $\mathrm{~T}-53$ & D-36 & 49,4 & MOP001-T810.2,7 & $\begin{array}{l}\text { MIN } 480 \mathrm{c} \\
\text { MAX } 570 \mathrm{C}\end{array}$ & Cumple \\
\hline $\begin{array}{l}\text { Punto de inflamación } \\
\qquad(\mathrm{OC})\end{array}$ & ------ & $\mathrm{T}-48$ & D-92 & 304 & MOP001-T810.2,5 & MIN 232 & Cumple \\
\hline Punto de combustión (oC) & ------ & $\mathrm{T}-48$ & D-92 & 323 & ASTM D113 & N/A & Cumple \\
\hline Ductivilidad (cm) & $-x_{-}$ & $\mathrm{T}-51$ & D-113 & $100+$ & MOP001-T810.2,3 & MIN 50 & Cumple \\
\hline Viscosidad (Pa. s) & ------ & ------ & D-2171 & 250,171 & ASTM D2170 & $\begin{array}{l}\text { MIN } 160 \\
\text { MAX } 240\end{array}$ & Cumple \\
\hline
\end{tabular}

N/A: No aplica

componentes acumulando el plomo en contenedores, para su posterior gestión. Separación del polipropileno: se aisló el polipropileno de la batería, acumulándose para su comercialización a gestores de residuos. Fundición: el plomo obtenido de las baterías (Scrap) se fundió al horno rotativo con aditivos como carbón, viruta de hierro y sosa cáustica para ayudar al proceso reductor del plomo en el horno (sedimentación parcial las de partículas pesadas). Al finalizar el proceso se produce el vaciado del horno, para obtener el plomo que luego será refinado. Refinación: se realizó la limpieza del plomo metálico libre de óxidos y escorias residuales. El material separado después de extraer el plomo, es el residuo denominado Escoria.

\section{Método Marshall [8-9]}

Para el diseño de las mezclas asfálticas en caliente se utilizó el método Marshall a través del análisis de estabilidad como fluencia, densidad y vacíos. La metodología Marshall, aunque es una metodología antigua fue suficiente para analizar el comportamiento en una mezcla asfáltica. El porcentaje óptimo de asfalto teńrirn șe calculó a partir de las siguientes fórmulas: $\% \mathbf{P}=\mathbf{M} * \mathbf{S}^{\frac{1}{5}}$, $\mathbf{S}$ se determina mediante $\mathbf{S}=\mathbf{0 , 1 7 G}+\mathbf{0 , 3 3 g}+\mathbf{2 , 3 A}+\mathbf{1 2}$ a + 135f. Dónde: $G=100$ - Porcentaje de mezcla tamiz № $3 / 8, \mathrm{~g}=$ Porcentaje de mezcla tamiz № 3/8 - Porcentaje de mezcla el tamiz № 4, A = Porcentaje de mezcla tamiz № 4 - Porcentaje de mezcla tamiz № 50, $a=$ Porcentaje de mezcla tamiz № 50 - Porcentaje de mezcla tamiz № 200,f = Porcentaje de mezcla tamiz № 200 y M= Varía 3,75 - 4,25. El valor obtenido fue de 5,39 \%. Para realizar la mezcla no modificada se partió con un valor de $6 \%$. El Instituto del Asfalto indica que se deben tomar dos porcentajes por encima y dos por debajo de cada 0,5. El ensayo Marshall se realizó utilizando porcentajes de asfalto de 5 $\%, 5,5 \%, 6 \%, 6,5 \%$ y $7 \%$. Se conformaron 3 briquetas para cada porcentaje, con un total de 15 especímenes. Para realizar la mezcla, los materiales se mantuvieron a una temperatura de $153^{\circ} \mathrm{C}$ a $158^{\circ} \mathrm{C}$; mientras que la temperatura de compactación fue de 142 oC a $147{ }^{\circ} \mathrm{C}$, obtenida de la carta de viscosidad, calculada a partir de la viscosidad del asfalto. Se realizó el ensayo de Gravedad Específica Bulk a las briquetas y se procedió con la rotura 
de las mismas. Posteriormente se disgregaron y se realizó el ensayo Rice (Norma: AASHTO T-209, ASTM D-2041). Una vez efectuado el ensayo Marshall, se realizaron los cálculos para obtener las propiedades mecánicas; Estabilidad y Flujo; y las propiedades volumétricas; Va, VAM y VAF. Finalmente, mediante la curva Porcentaje de Asfalto vs. Estabilidad se obtuvo un porcentaje óptimo de asfalto de $6,5 \%$

\section{Diseño de mezcla asfáltica modificada}

Se tamizaron los desechos especiales con el fin de excluir los pedazos sobrantes del proceso de trituración de la escoria, se realizó el proceso de tamizado utilizando el tamiz \# 4. Con el material tamizado se realizó el reemplazo en la mezcla original correspondiente al peso acumulado en el tamiz \#8, en porcentajes de escoria de $5 \%, 15 \%$, $25 \%$ y $35 \%$, en relación al agregado fino. Los desechos especiales (Escoria) proveniente de baterías desechadas de vehículos presentó un porcentaje de absorción de agua de $13 \%$, este resultado dificulta la caracterización de la escoria debido a que tiende a reaccionar con el agua y en casos de precipitación se presentarán problemas de reacción, lixiviación, infiltración, favorables a la formación de grumos de material. De igual manera en la mezcla con los agregados y el ligante asfáltico mientras mayor porcentaje de escoria se añada se absorbe el asfalto y dificulta que la mezcla homogénea sea compactada. Por lo antes expuesto, la escoria debe añadir en el momento de la mezcla y no mezclarla con los agregados sin someter al horno, para evitar pérdidas por evaporación y formación de grumos indeseables.

\section{Módulo de rigidez}

Para determinar el comportamiento del pavimento ante la carga vehicular se determinó el Módulo de rigidez [10], el cual es un método no destructivo de tracción indirecta. En este ensayo se utilizó una Máquina Universal marca Cooper HYD25. Se proporcionaron pulsos de cargas con períodos de descanso en forma repetitiva, se ensayaron dos briquetas para mezcla no modificada y dos briquetas para mezcla modificada. Las muestras ensayadas se llevaron a una temperatura de $25 \pm 1{ }^{\circ} \mathrm{C}$ y se centraron dentro del bastidor de montaje. Se ajustaron los deformímetros y la posición de la célula de carga hasta llegar a la posición de ensayo.

\section{Resultados y Discusión}

La mezcla modificada por vía seca, con desechos especiales (Escoria) proveniente de baterías desechadas de automóviles $(6,5 \%$ de asfalto óptimo), fue analizada con especificaciones de la norma MOP 001 2002, correspondiente a tráfico muy pesado. En comparación con su respectiva mezcla convencional, el aglomerante óptimo en la mezcla modificada tiende a aumentar, lo cual se atribuyó al área de superficie lisa de partículas finas de escoria. El aumento del porcentaje de escoria en la mezcla, produjo que la densidad Bulk en la briqueta disminuya (Figura 1a). Para la mezcla asfáltica con el 5\% de escoria se obtuvo una densidad Bulk de 2,276 $\left(\mathrm{gr} / \mathrm{cm}^{3}\right)$ y para ese mismo porcentaje se una estabilidad de 4311 (lbf), Figura 1b; este último valor mayor al requerido por el MTOP para tráfico muy pesado (2200 (lbf)). De acuerdo a los estudios realizados, el $5 \%$ de escoria en la mezcla es el más adecuado, y a pesar de ser el porcentaje de escoria más bajo representa una mejora en las características mecánicas de la mezcla y un ahorro ambiental. El valor del flujo obtenido disminuye con el aumento de la proporción de escoria en la mezcla (Figura 1c). Para la mezcla asfáltica con el $5 \%$ de escoria se obtuvo un flujo de 14 centésimas de pulgada, valor dentro de los parámetros permitidos por la norma. Los vacíos (Va) dentro la mezcla asfáltica modificada tienden a aumentar con el porcentaje de escoria (Figura 2a). Incorporando un porcentaje de $5 \%$ de escoria, los Va pueden mantenerse en un valor aceptable por la norma. Los VAM en las mezclas modificadas se mantienen contaste entre un $5 \%$ y $15 \%$ de escoria (Figura $2 \mathrm{~b}$ ), aumentaron a partir de $15 \%$ y posterior a este valor se obtuvo valores superiores a los sugeridos en la norma. Para la mezcla asfáltica con el $5 \%$ de escoria se tiene un valor de $15 \%$, valor aceptable dentro del requerido por la norma. Los VAF disminuyen con el aumento en el porcentaje de escoria en la mezcla (Figura 2c). Para la mezcla asfáltica con el $5 \%$ de escoria se tiene un VAF equivalente a $75,04 \%$, valor que se encuentra dentro de los límites permitidos ( $65 \%$ a $75 \%$ ). Mediante los resultados obtenidos se sugiere que el porcentaje óptimo de escoria que se debe considerar para la mezcla es del $5 \%$.

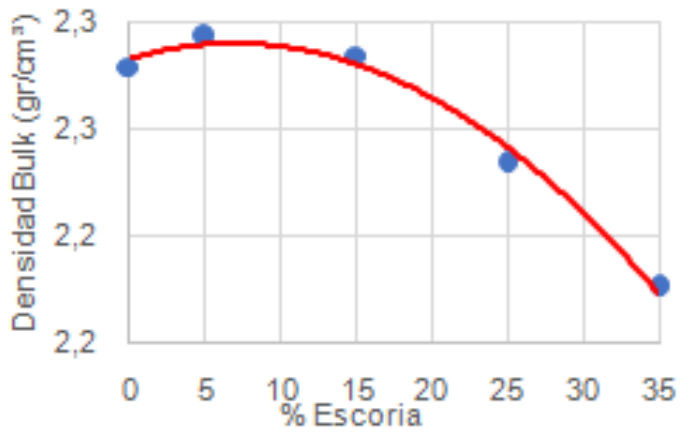

(a)

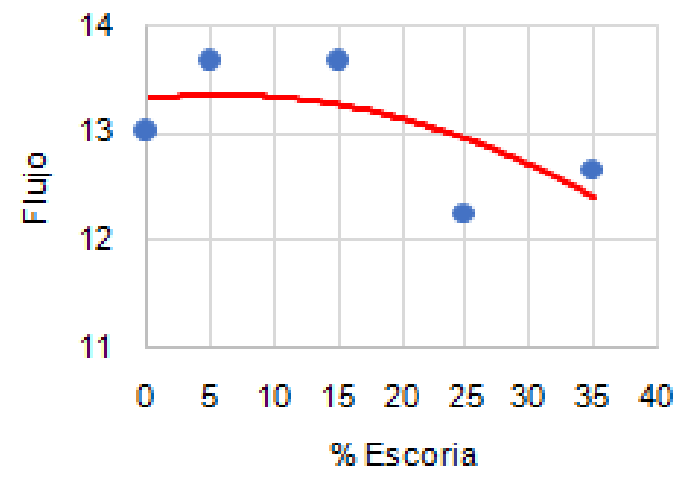

(b) 


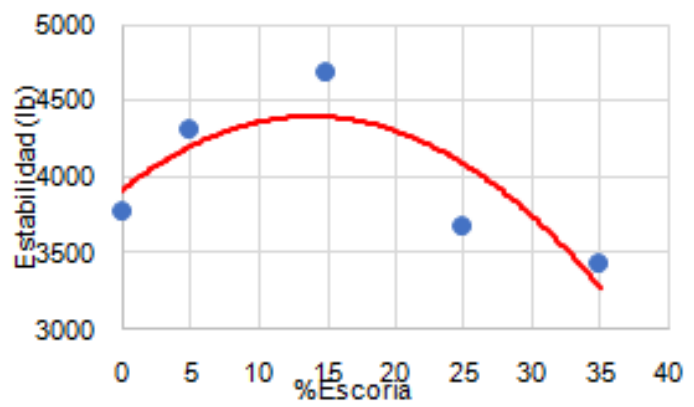

(c)

Figura 1: a) \% Escoria vs. Densidad Blk, b) \%Escoria vs. Estabilidad, c) \%Escoria vs. Flujo



(a)



(b)

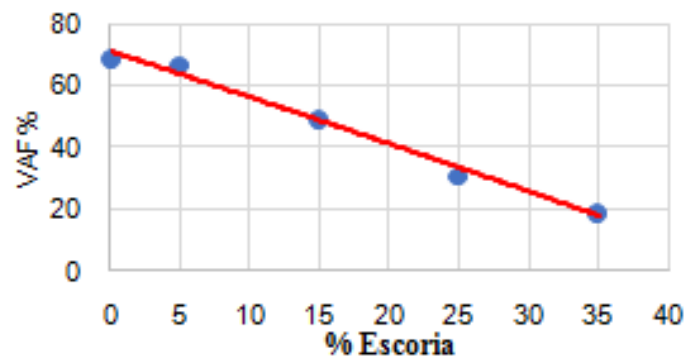

(c)

Figura 2. a) \% Escoria vs. Va, b) \% Escoria vs. VAM y c) $\%$ Escoria vs. VAF
En la Tabla 3, se resume y comparan los resultados obtenido en el ensayo Marshall utilizando la mezcla no modificada y la modificada. La densidad Bulk, permite verificar la efectividad del proceso de compactación que se lleva acabo para conformar la mezcla, cuando se compararan los resultados entre las mezclas ensayadas se obtuvo que la compatibilidad se mantiene. En comparación con la mezcla sin modificar, la muestra modificada presenta un aumento de un $30 \%$ en estabilidad y un $8 \%$ en flujo. La estabilidad, es la resistencia que puede tener la mezcla a la deformación frente a una carga externa, mientras que el flujo es la deformación que presenta la briqueta. Mediante varios ensayos de laboratorio y diferentes muestras ensayadas, se puede definir que una mezcla con valores bajos de fluencia y altos de estabilidad son mezclas frágiles y rígidas, por otra parte, se considera mezclas plásticas $\mathrm{y}$ tienden a deformarse con facilidad bajo cargas externas a mezclas con altos valores de fluencia. Los resultados obtenidos, predicen una mejora en las propiedades mecánicas de la mezcla modificada en cuanto a aumento en resistencia a medida que se aumentó el porcentaje de escoria en la mezcla. Por otro lado, la relación entre la estabilidad de Marshall y el valor de flujo, es un indicador de la resistencia del material a la deformación [11], según las especificaciones de MoRT \& H 2013, este valor debe estar entre 2 y 5 . La mezcla modificada cumplió con este criterio requerido, lo que sugiere una mejor resistencia contra las grietas bajo una fuerte rigidez en el movimiento del tráfico.

Obtener el porcentaje de huecos vacíos y rellenos de las briquetas, permite llevar un control base de compactación en la construcción del pavimento asfáltico. Las bolsas de aire formadas en una mezcla asfáltica compactada entre el agregado y el asfalto son los vacíos que definen la calidad de la mezcla y su utilización en la estructura del pavimento. Dentro de estos vacíos están un porcentaje de huecos formados por las partículas del agregado pétreo, el VAM, el resto de los vacíos están llenos de asfalto y se lo define como VFA. En general, en la presente investigación cuando se modificó la mezcla todos los tipos de porcentaje de vacíos disminuyeron, lo que se asocia al incremento de la cantidad de material fino dado que la fracción de material escoria utilizada corresponde al material pasante el tamiz No.8, definido como un elemento de partículas finas. Del análisis de la granulometría realizada a los agregados pétreos y porcentaje de vacíos de la mezcla asfáltica modificada, resulta una mezcla asfáltica modificada cerrada. De acuerdo a este resultado, se recomienda la mezcla útil como capa de rodadura en la estructura del pavimento flexible, la cual se diseña para resistir la abrasión y desintegración por efectos ambientales. Por los ensayos realizados a las briquetas se pudo observar la reacción que tenía la mezcla asfáltica modificada por vía seca con el agua, por lo cual se sugiere que la capa de rodadura se diseñe para lugares que no tengan grandes intensidades de lluvia, conjuntamente con un análisis químico del agua que está en contacto con la capa de rodadura. 
Tabla 3. Resume y comparación de los resultados obtenidos en el ensayo Marshall.

Según la norma MOP 001-T 405.5.4.

\begin{tabular}{|c|c|c|c|c|c|}
\hline \multirow{2}{*}{ Parámetro } & \multirow{2}{*}{$\begin{array}{c}\text { Mezcla original: } 6,5 \% \\
\text { de asfalto }\end{array}$} & \multirow{2}{*}{$\begin{array}{c}\text { Mezcla: } 6,5 \% \\
\text { de asfalto y } 5 \% \\
\text { escoria }\end{array}$} & \multicolumn{2}{|c|}{ Trafico muy pesado } & \multirow{2}{*}{ Verificación } \\
\hline & & & MÍN & MÁX & \\
\hline Densidad Bulk g/ $\mathrm{cm}^{3}$ & 2,245 & 2,276 & --- & --- & Cumple \\
\hline Estabilidad (lb) & 3312 & 5311 & 2200 & ---- & Cumple \\
\hline Flujo $0,01 "$ & 13 & 14 & 8 & 14 & Cumple \\
\hline $\mathrm{Va}(\%)$ & 5,5 & 4,6 & 3 & 5 & Cumple \\
\hline VAM (\%) & 15 & 14 & 14 & ---- & Cumple \\
\hline VAF (\%) & 75,04 & 67 & 65 & 75 & Cumple \\
\hline
\end{tabular}

\section{Ensayo de módulo de rigidez}

Los ensayos para el módulo de rigidez efectivo a la tracción indirecta, resultaron en un aumentó de 825,7 Mpa en la mezcla sin modificar a 1667,8 Mpa en la mezcla modificada, y la deformación horizontal se redujo de 6,26 $\mu \mathrm{m}$ a $4,67 \mu \mathrm{m}$. Estos valores son indicadores potenciales del agrietamiento que puede sufrir el pavimento, una deformación alta a la falla, orientan si la mezcla asfáltica puede tolerar deformaciones altas antes de fallar, lo que significa que probablemente resista más el agrietamiento que una mezcla con una deformación baja a la falla [12]. La mezcla modificada presenta mayor rigidez y menor deformación en comparación con la mezcla sin modificar.

\section{Conclusiones}

El diseño de la mezcla asfáltica modificada mediante el método Marshall, y el posterior control de calidad realizado conforme las especificaciones técnicas correspondientes del MTOP, determinó que la calidad de ésta es satisfactoria. Los parámetros de granulometría del agregado mineral, porcentaje de vacíos, vacíos en el agregado mineral (VAM) y vacíos rellenos de asfalto (VFA) se mantienen en los rangos requeridos en las especificaciones técnicas del MTOP, por tanto, los ensayos pueden ser considerados adecuados como criterio de aceptación de la mezcla asfáltica modificada. La estabilidad y el flujo obtenido para las mezclas modificadas con escoria, presentan un incremento con relación a los valores obtenidos en briquetas sin modificar, por lo que los ensayos realizados a la mezcla sin modificar pueden considerarse como adecuados para definir la aceptación de una mezcla asfáltica. De acuerdo a este resultado, se considera la mezcla útil como capa de rodadura en la estructura del pavimento flexible, la cual se diseña para resistir la abrasión y desintegración por efectos ambientales. Este tipo de mezcla se recomienda para ser utilizada en lugares de poca precipitación, ya que solo el análisis fue por vía seca; en un futuro se pretende realizar ensayos por vía húmeda para evaluar cuál es su comportamiento.

\section{Agradecimientos}

Los autores agradecen al Ing. Jorge Bucheli del Laboratorio de Materiales de Construcción (PUCE-Quito, Ecuador) por su invaluable asistencia técnica. A planta Asfáltica Naranjo Ordoñez, Quito-Ecuador por haber proporcionado el material.

\section{Referencias Bibliográficas}

[1] Castells X. E.: Reciclaje y tratamiento de residuos diversos: Reciclaje de residuos industriales. Ediciones Díaz de Santos, 2012.

[2] Díaz J. C. and Arias M. L. D.: La contaminación por pilas y baterías en México. Gaceta Ecológica, Vol. 72, (2004) 53-74.

[3] De Aburrá, Á. M. D. V. y Universidad Pontificia Bolivariana (UPB): Guía para el Manejo Integral de Residuos. Area Metropolitana del Valle del Aburra, 2008.

[4] Ochoa G. S.: Revisión y análisis de las experiencias de Argentina, Brasil, Colombia, Ecuador y México respecto de los cinco elementos claves para el manejo ambiental de pilas y baterías, 2001.

[5] González S. F., Couce L. C., Guerreiro M. J. R., Formoso, J. Á. F. and Villa, R. Estudio, análisis y valorización de residuos industriales procedentes de la industria de la construcción naval, para su reciclaje y uso en otras aplicaciones marinas, 2000.

[6] Cárdenas J. y Fonseca E.: Modelación del comportamiento reológico de asfalto convencional y modificado con polímero reciclado, estudiada desde la relación viscosidad-temperatura (modeling of rheological behavior of conventional and recycled polymer modified asphalt. Revista EIA, Vol. 6, No. 12 (2013)125-137. 
[7] Iturbide J. C.: Manual centroamericano para diseño de pavimentos. SIECA. Guatemala, 2002.

[8] Garnica Anguas P., Delgado Alamilla H. y Sandoval Sandoval C. D.: Análisis comparativo de los métodos Marshall y Superpave para compactación de mezclas asfálticas. Publicación técnica, (271), 2005)

[9] Garnica Anguas P., Flores Flores M., Gómez López J. A. y Delgado Alamilla H.: Caracterización geomecánica de mezclas asfálticas. Publicación técnica, (267), 2005.

[10] Reyes-Ortiz O. J. y Rincón J. F.: Influencia de las características del relleno mineral en la resistencia de una mezcla asfáltica. Revista EIA, 2013.
[11] Hattatoglu F., Hinislioglu S., Bayata H., Ünsal Bayrak O., N. T., F. Hattatoglu, 1st international conference on engineering and natural sciences, in: Ö.H.H.S.-K. Çinar (Ed.), Modeling of Marshall Quotient of Hot Mix Asphalts by Artificial Neural Networks, 2015, pp. 4-11.

[12] Flores, M \& Garnica, P \& Gómez, J \& Delgado, H. (2005). Caracterización geomecánica de mezclas asfálticas. Sanfandilla. Publicación técnica No. 267.Recuperadode:http://www.imt.mx/archivos/ publicaciones/publicaciontecnica/pt267.pdf. 


\section{REVISTA TECNICA}

DE LA FACULTAD DE INGENIERIA

UNIVERSIDAD DEL ZULIA

Vol. 43. N`1, Enero - Abril 2020, pp. 03 - 56

Esta revista fue editada en formato digital y publicada en Diciembre de 2019, por el Fondo Editorial Serbiluz, Universidad del Zulia. Maracaibo-Venezuela

www.luz.edu.ve

www.serbi.luz.edu.ve

www.produccioncientifica.luz.edu.ve 